\title{
Time-Course Changes in Left Ventricular Geometry and Function during the Development of Hypertension in Dahl Salt-Sensitive Rats
}

\author{
Peng QU, Mareomi HAMADA, Shuntaro IKEDA, Go HIASA, Yuji SHIGEMATSU, \\ and Kunio HIWADA
}

\begin{abstract}
Serial changes of left ventricular (LV) geometry and function during the development of hypertension were studied in 50 Dahl salt-resistant (DR) and 88 Dahl salt-sensitive (DS) rats fed an $8 \% \mathrm{NaCl}$ diet beginning at the age of 6 weeks. Echocardiography at $6,8,11,13,14,15$, and 18 weeks and in vivo invasive hemodynamic determination at $6,8,11,14$, and 18 weeks were performed. After 11 weeks, 33 DS rats were observed for survival analysis. The survival analyses showed that the incidence of death was $57.6 \%$ due to heart failure, $\mathbf{2 7 . 3} \%$ due to stroke, and $\mathbf{1 5 . 2} \%$ due to sudden death. However, death in the early stages of hypertension was due almost entirely to sudden death or stroke. A high value of relative wall thickness (RWT) and a small end-diastolic dimension were predictive of sudden death and stroke, but LV mass (LVM) was not. Concerning the change in LV geometric patterns, LVM continued to increase to 18 weeks. The RWT increased or remained at a plateau up to 13 weeks, and then progressively decreased after 13 weeks. In contrast, LV function was hyperdynamic between 8 and 11 weeks when compared to DR rats. However, after 13 weeks, all hemodynamic variables of DS rats deteriorated progressively, and all DS rats died of heart failure. Thus, our study indicates that the cardiovascular events associated with the progression of hypertension vary widely according to the stage of hypertension and that RWT is more sensitive in predicting LV conditions than LVM in hypertension. (Hypertens Res 2000; 23: 613-623)
\end{abstract}

Key Words: hypertension, left ventricular geometry, heart failure, sudden death, stroke, Dahl rat

\section{Introduction}

Left ventricular (LV) hypertrophy predicts the prognosis of hypertensive cardiovascular events independent of arterial pressure or other risk factors $(1,2)$. Furthermore, LV geometric changes and deterioration of left ventricular function also predict the subsequent morbidity and mortality of cardiovascular events and extracardiac targetorgan damage (1-5). Thus, serial observations of LV geometry and functional changes from the development of hypertension to heart failure should be emphasized. However, it is very difficult to document these changes in hypertensive patients due to the difficulty of performing the longitudinal study itself, excluding obstructive coronary artery disease, age-related changes in cardiac structure, and so on as causes of deterioration in LV function.

Many experimental models of heart failure have been studied extensively (6-9). However, only a few models have clearly demonstrated a transition from compensatory hypertrophy to decompensated heart failure (10-12). The Dahl salt-sensitive (DS) rat is thought to be an appropriate model for investigating the characteristics of myocardial hypertrophy and its transition to heart failure (13). Since the pressure rise in the Dahl rat is gradual, the LV mass increases in response to greater physiological load,

From the Second Department of Internal Medicine, Ehime University School of Medicine, Ehime, Japan.

Address for Reprints: Mareomi Hamada, M.D., Second Department of Internal Medicine, Ehime University School of Medicine, Shigenobu-cho, Onsen-gun, Ehime 791-0295, Japan.

Received February 7, 2000; Accepted in revised form June 6, 2000. 
resulting in a limited lifespan, are more similar to human cardiac hypertrophy than hypertension in a surgically induced model. Previous studies have shown that most DS rats fed a high-salt diet after weaning have a life expectancy of less than 20 weeks (13-15), with the primary cause of death has been heart failure (13). The heart in this model is characterized by significant concentric hypertrophy and a hyperdynamic condition at 11 weeks of age and by subsequent decompensation with dilated hypokinetic LV after 16 weeks of age (13). The survival data, however, were not consistent (13-16), and the relation between LV geometric changes and cardiovascular events remains to be determined in this model.

Thus, the purpose of the present study was 1) to document the in vivo evolution of hypertension and the transition to heart failure in salt-loading Dahl rats, 2) to establish the changes in LV geometry and LV function in this process, and 3) to assess the prognostic significance of LV geometry in DS rats.

\section{Methods}

\section{Animal Models}

Fifty male Dahl salt-resistant (DR) rats and 88 male DS rats (Japan SLC. Inc., Hamamatsu, Japan), all of which were 4-5 weeks old at the beginning of the study, were housed 2 rats per cage under controlled conditions of light, temperature and humidity. The rats were fed a $0.3 \% \mathrm{NaCl}$ (low-salt) diet until the age of 6 weeks, after which time they were fed an $8 \% \mathrm{NaCl}$ (high-salt) diet (MF rat diet, Oriental Yeast Industry, Chiba, Japan) and tap water ad libitum.

Systemic blood pressure and body weight were measured once a week. Peak systolic pressure was recorded by the tail-cuff method (Model PS-100, Riken Kaihatsu, Yokohama, Japan). Values for systolic pressure were defined by averaging measurements repeated five times.

\section{Protocol 1}

Thirty-three DS rats were monitored for survival data. After the age of 11 weeks the rats were kept under close observation, as sudden death and stroke often are common in these rats. The symptomatic stroke was diagnosed by clinical standards, including body paralysis, convulsions, and disordered movement. Rats for whom death had been detected were autopsied within $2 \mathrm{~h}$, and the cranial cavity was examined to macroscopically determine the type of stroke that had occurred. Heart failure was diagnosed by clinical definitions, serial echocardiographic observations, and LV catheterization. The DS rat suffering from heart failure was characterized by loss of body weight, fatigue, reductions in movement, tearing, and finally, rapid and labored respiration. At this stage the blood pressure tended to decrease. The rats often died within 1 week of the onset of dyspnea.

\section{Protocol 2}

Serial echocardiographic documentations and LV catheterization were performed in $55 \mathrm{DS}$ rats and $50 \mathrm{DR}$ rats. After catheterization, the rats were sacrificed by the drawing of blood, after which the cardiac atria, cardiac ventricles, lungs, and kidneys were carefully resected and weighed.

\section{Echocardiography}

To elucidate the in vivo LV geometry and contractile function in rats, transthoracic echocardiography was performed at ages $6,8,11,13,14,15$, and 18 weeks (model SSH-140A, Toshiba, Tokyo, Japan), with a $7.5-\mathrm{MHz}$ sector scan probe. In addition, for DS rats the final echocardiogram was taken just before death when labored respiration became apparent. Each rat was lightly anesthetized with $15 \mathrm{mg} / \mathrm{kg}$ of i.p. pentobarbital, and the left chest was shaved. Animals were then placed in the left lateral decubitus position. In failure rats with severe fatigue, anesthesia was not needed. Using a transducer focused on $7 \mathrm{~mm}, \mathrm{M}$-mode echocardiograms at the papillary muscle level were obtained, guided by two-dimensional short-axis views, and were recorded on a recorder (model TP 8700, Toshiba, Tokyo, Japan) at a paper speed of 100 $\mathrm{mm} / \mathrm{s}$. The LV dimension was determined by the leading edge method from five consecutive cardiac cycles according to the modification of the American Society for Echocardiography (17). The LV end-diastolic dimension (EDD) and the end-systolic dimension (ESD) were measured at the widest and the narrowest dimensions for $\mathrm{M}$ mode recording, respectively. The LV posterior wall thickness at end-diastole and end-systole (PWTd and PWTs) was measured at EDD and ESD. The relative wall thickness (RWT) was measured as $2 \times P W T d / E D D$. The changing of posterior wall thickness (\%PWT) between end-systole and end-diastole was also assessed as follows: $\% \mathrm{PWT}=(\mathrm{PWTs}-\mathrm{PWTd}) / \mathrm{PWTd} \times 100$. End-diastolic volume (EDV) and end-systolic volume were calculated from EDD and ESD, and the ejection fraction (EF) was measured as (EDV-ESV)/EDV $\times 100$.

The LV mass was calculated using a standard cube formula $(18,19) . \mathrm{LV}$ mass $(\mathrm{g})=1.04 \times\left\{[\mathrm{EDD}+(2 \times \mathrm{PWTd})]^{3}\right.$ $-\mathrm{EDD}^{3\}}$, where 1.04 is the specific gravity of muscle. A regression equation was used to compared the calculated $\mathrm{LV}$ mass to postmortem LV mass in DR and DS rats $(y=-0.14+1.29 x, r=0.91, p<0.0001)$ (Fig. 1). The modified equation, LV mass=cube formula mass $\times$ $0.78+0.11$, was then prospectively applied to subsequent echocardiographic examinations.

LV endocardial fractional shortening (FS) was calcu- 


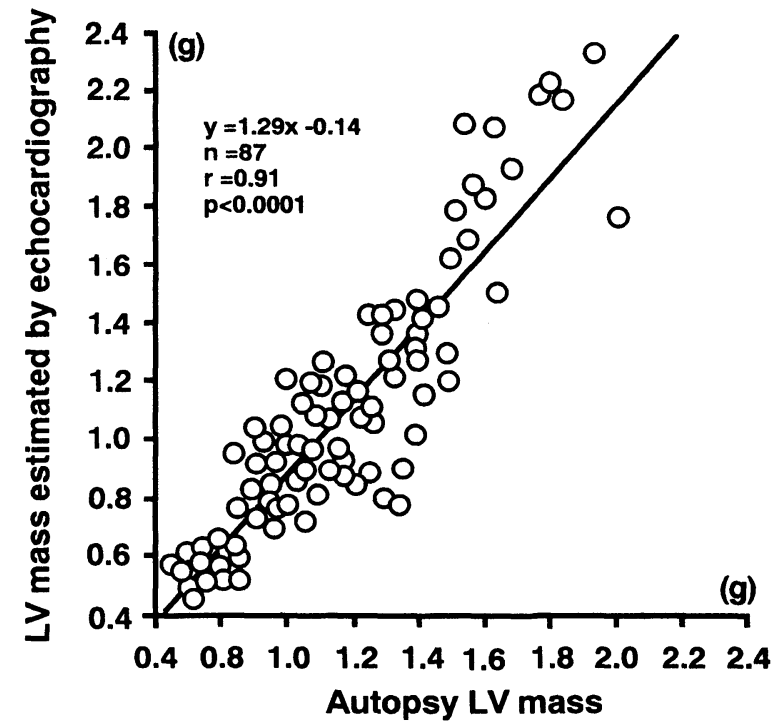

Fig. 1. The relationship between $L V$ mass estimated by echocardiography and corresponding postmortem LV mass $(\mathrm{n}=87 ; 47$ every stage of Dahl salt-sensitive rats and $40 \mathrm{ev}$ ery stage of Dahl salt-resistant rats).

lated as $\mathrm{FS}(\%)=[(\mathrm{EDD}-\mathrm{ESD}) / \mathrm{EDD}] \times 100$. In addition, since the inner half of the left ventricular wall contributes more to total left wall thickening than does the outer half, midwall fractional shortening (mFS) was also calculated according to the two-shell cylindrical model (20) as follows:

$$
\begin{aligned}
\operatorname{mFS}(\%)= & \{(\mathrm{EDD}+\mathrm{PWTd})-(\mathrm{ESD}+\mathrm{PWTs})\} /(\mathrm{EDD} \\
& +\mathrm{PWTd}) \times 100 .
\end{aligned}
$$

\section{Hemodynamic Determination}

In DS rats at $6,8,11,14$, and 18 weeks of age or just before death by heart failure, the invasive pressures of the carotid artery and left ventricle were recorded after the echocardiographic study. Ten rats were recorded at each time point. Age-matched DR rats were also examined. Animals were anesthetized with sodium pentobarbital (50 $\mathrm{mg} / \mathrm{kg}$, administered intraperitoneally), and if the anesthetic condition was unstable, a further $10 \mathrm{mg} / \mathrm{kg}$ of sodium pentobarbital was given. Each rat was placed on its back and was allowed to breathe spontaneously. A catheter pressure transducer (Model SPC-320, Millar, Houston, USA) was inserted through the right carotid artery into the left ventricle. Zero level was calibrated electrically with a transducer control unit (Model TC-510; Millar) before insertion into the artery. The first derivative of the left ventricular pressure curve $(\mathrm{d} P / \mathrm{d} t)$ was obtained by electrical differentiation of the pressure signal. Hemodynamic parameters were recorded on a multichannel recorder (model WS-682G; Nihon Kohden,
Tokyo, Japan) at a paper speed of $200 \mathrm{~mm} / \mathrm{s}$. Using pressure recordings, left ventricular peak systolic and end diastolic pressures were measured, and the $+\mathrm{d} P / \mathrm{d} t$ and the $-\mathrm{d} P / \mathrm{d} t$ were obtained from the first derivative of the left ventricular pressure. The $\mathrm{d} P / \mathrm{d} t / P$ was also calculated. All hemodynamic parameters were averaged for five consecutive beats.

\section{Estimation of LV Wall Stress}

Circumferential and meridional end-systolic wall stresses at midwall (ESSmwc and ESSmwm), used as indices of myocardial afterload, were calculated using a cylindrical model that did not require direct measurement of the LV long axis (21-23). Systolic blood pressure (SBP) measured by the tail cuff method was used to calculate the midwall stress. ESSmwc and ESSmwm were calculated as follows:

$$
\begin{aligned}
\mathrm{ESSmwc}= & \mathrm{SBP} \times(\mathrm{ESD} / 2)^{2} \times\left[1+(\mathrm{ESD} / 2+\mathrm{PWTs})^{2}\right] / \\
& \left\{(\mathrm{ESD} / 2+\mathrm{PWTs} / 2)^{2} \times\left[(\mathrm{ESD} / 2+\mathrm{PWTs})^{2}\right.\right. \\
& \left.\left.-(\mathrm{ESD} / 2)^{2}\right]\right\} . \\
\mathrm{ESSmwm}= & 0.334 \times \mathrm{SBP} \times(\mathrm{ESD}+\mathrm{PWTs}) / \mathrm{PWTs} /(\mathrm{ESD} \\
& +2 \mathrm{PWTs}) .
\end{aligned}
$$

\section{Statistical Analysis}

All values are shown as mean \pm SEM. The primary factors (group, time, and interaction of group and time) were tested using a two-factor ANOVA for repeated measures followed by Fisher's protected least significant difference test for between-group comparisons. Differences at specific time points (between groups and within groups) were assessed using one-factor ANOVA with post hoc comparisons by Fisher's protected least significant difference test. Correlation coefficients were obtained using linear regression (the method of least squares). The survival analysis was performed by Kaplan-Meier analysis. A probability of $<0.05$ was considered significant.

\section{Results}

\section{Serial Changes in Systolic Blood Pressure and LV Mass}

Figure 2 shows the serial changes in systolic blood pressure (A), body weights (B) and left ventricular weight/ body weight $(\mathrm{LVW} / \mathrm{BW})$ ratios $(\mathrm{C})$ of $\mathrm{DR}$ and $\mathrm{DS}$ rats from ages 6 to 18 weeks. As shown in Fig. 2A, the blood pressures in DS rats progressively increased, reaching 250 $\mathrm{mmHg}$ by 12 weeks, reached a plateau at 13 weeks, and then began to decrease. In contrast, blood pressure in DR rats remained unchanged during these periods. As shown in Fig. 2B, body weight decreased in DS rats after the age of 13 weeks, whereas that in DR rats continued to increase. As shown in Fig. $2 \mathrm{C}$, the left ventricular LVW/BW ratio in DS rats gradually increased, while that 

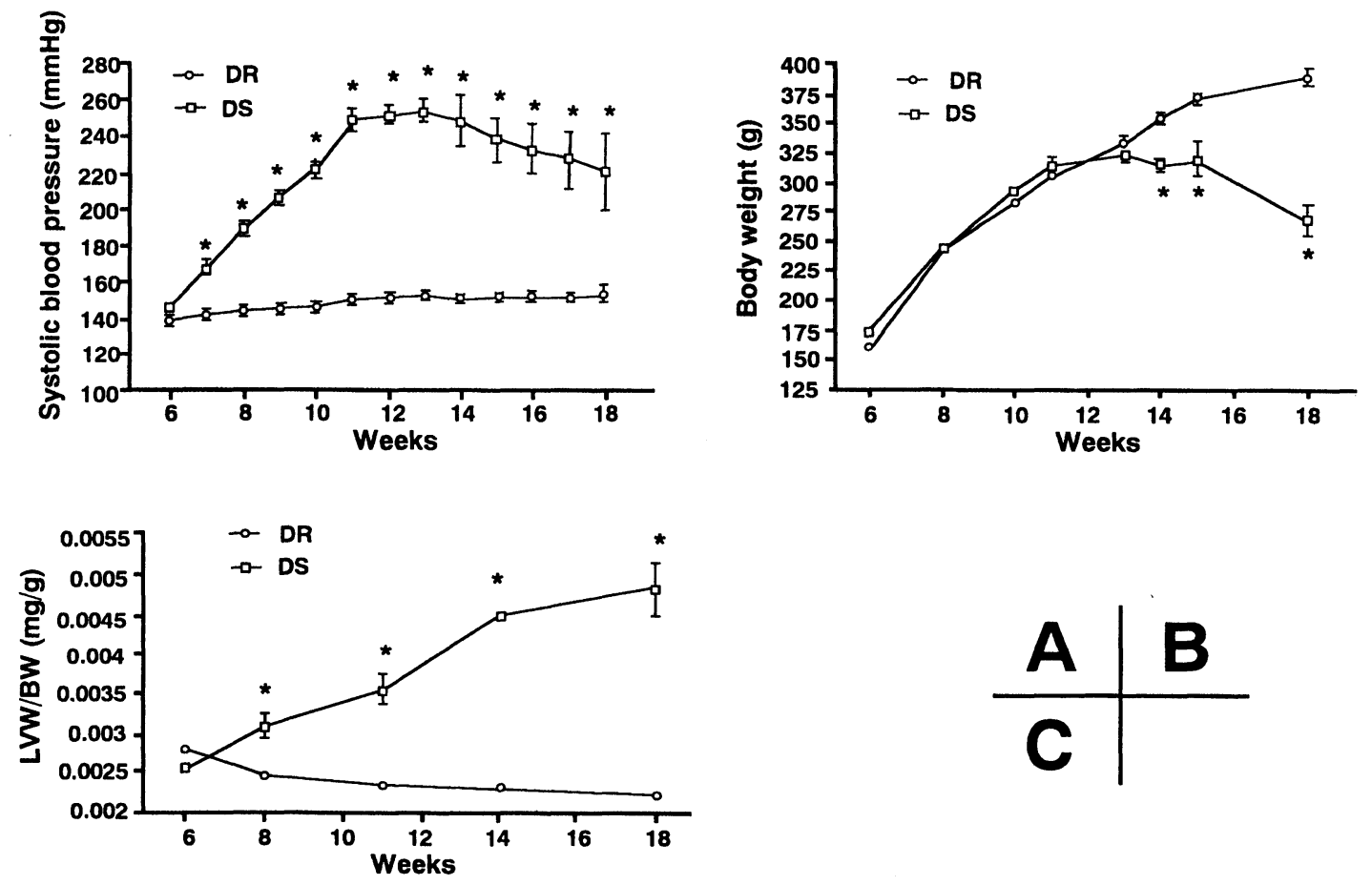

Fig. 2. A: Time course of blood pressure in Dahl salt-resistant (DR) and Dahl salt-sensitive (DS) rats after salt-loading, $B$ : time courses of body weight change in DR and DS rats, C: left ventricular weight normalized for body weight $(\mathrm{LVW} / \mathrm{BW})$ taken at the ages of $6,8,11,14$, and 18 weeks in $D R(\mathrm{n}=20)$ and $D S$ rats $(\mathrm{n}=18)$ fed an $8 \% \mathrm{NaCl}$ diet. $* \mathrm{p}<$ 0.01 vs. $D R$.

Table 1. Cumulative Survival Analysis, the Mortality of Heart Failure and the Incidence of Symptomatic Stroke in DS Rats after $8 \%$ Salt Diet

\begin{tabular}{|c|c|c|c|c|c|c|c|c|}
\hline \multirow{2}{*}{ Weeks } & \multicolumn{2}{|c|}{ Cumulative survival } & \multicolumn{2}{|c|}{ Cumulative sudden death } & \multicolumn{2}{|c|}{ Cumulative heart failure } & \multicolumn{2}{|c|}{ Cumulative stroke } \\
\hline & Rate $(\%)$ & $n$ & Incidence $(\%)$ & $n$ & Incidence $(\%)$ & $n$ & Incidence $(\%)$ & $n$ \\
\hline 11 & 100.0 & 33 & 0.0 & 0 & 0.0 & 0 & 0.0 & 0 \\
\hline 11.5 & 78.8 & 26 & 15.1 & 5 & 0.0 & 0 & 6.1 & 2 \\
\hline 12 & 75.8 & 25 & 15.1 & 5 & 0.0 & 0 & 9.1 & 3 \\
\hline 13 & 63.6 & 21 & 15.1 & 5 & 3.0 & 1 & 18.2 & 6 \\
\hline 14 & 33.3 & 11 & 15.1 & 5 & 24.2 & 8 & 27.3 & 9 \\
\hline 15 & 27.3 & 9 & 15.1 & 5 & 30.3 & 10 & 27.3 & 9 \\
\hline 16 & 24.2 & 8 & 15.1 & 5 & 33.3 & 11 & 27.3 & 9 \\
\hline 17 & 21.2 & 7 & 15.1 & 5 & 36.4 & 12 & 27.3 & 9 \\
\hline 18 & 6.1 & 2 & 15.1 & 5 & 51.5 & 17 & 27.3 & 9 \\
\hline 19 & 3.0 & 1 & 15.1 & 5 & 54.5 & 18 & 27.3 & 9 \\
\hline 20 & 0.0 & 0 & 15.1 & 5 & 57.6 & 19 & 27.3 & 9 \\
\hline
\end{tabular}

in DR rats remained almost constant.

\section{Survival Analysis of DS Rats}

Table 1 and Fig. 3 show an analysis of cumulative survival and the incidence of sudden death, heart failure, or symptomatic stroke in DS rats. Symptomatic stroke began to occur at an age of 11.5 weeks. DS rats most often died of stroke before 13 weeks. Sudden death was observed in five rats. Of these five, two were confirmed to have cerebral hemorrhage by macroscopic observation, while the other three showed no evidence of heart failure, cerebral hemorrhage, or infarction. Heart failure began to occur after the age of 13 weeks, and DS rats usually died of 
heart failure after 14 weeks.

\section{Comparison of LV Geometric Parameters and Systol- ic Blood Pressures among Three Types of Car- diovascular Events in DS Rats}

Table 2 provides a comparison of LV geometric parameters and systolic blood pressures at 11 weeks of age among DS rats with symptomatic stroke, early-developed heart failure before the age of 15 weeks, sudden death, or late-developed heart failure after the age of 15 weeks. Age-matched DR rats are also shown as controls. The PWTd and PWTs, LV mass, and systolic blood pressure were greater in each group of DS rats than in the DR rats. In contrast, both EDD and ESD were smaller in DS

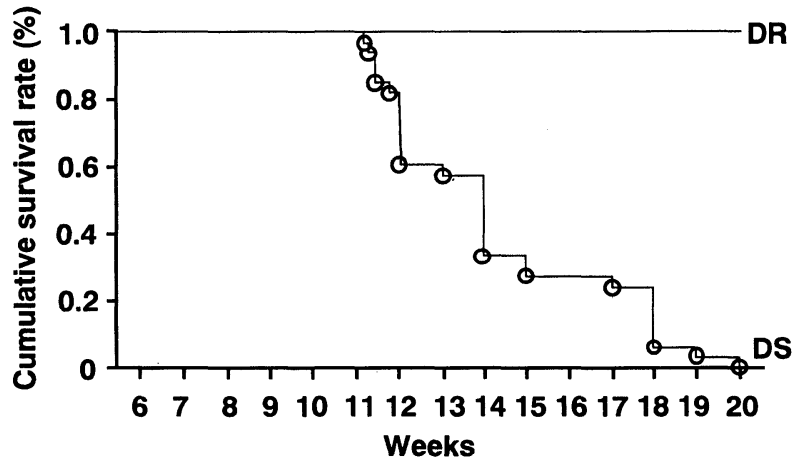

Fig. 3. The cumulative survival curve of Dahl salt-resistant (DR) and salt-sensitive (DS) rats after $8 \%$ salt diet.

Table 2. Comparisons of LV Geometric Parameters and Systolic Blood Pressure at Age of 11 Weeks among Three Types of Cardiovascular Events in DS Rats

\begin{tabular}{lrllllllc}
\hline & $n$ & $\begin{array}{c}\text { PWTd } \\
(\mathrm{mm})\end{array}$ & $\begin{array}{l}\text { PWTs } \\
(\mathrm{mm})\end{array}$ & $\begin{array}{l}\text { EDD } \\
(\mathrm{mm})\end{array}$ & $\begin{array}{c}\text { ESD } \\
(\mathrm{mm})\end{array}$ & $\begin{array}{c}\text { RWT } \\
(2 \mathrm{PWTD} / \mathrm{EDD})\end{array}$ & $\begin{array}{c}\text { LVM } \\
(\mathrm{g})\end{array}$ & $\begin{array}{c}\text { SBP } \\
(\mathrm{mmHg})\end{array}$ \\
\hline DR & 21 & $1.8 \pm 0.1$ & $2.9 \pm 0.1$ & $7.4 \pm 0.1$ & $3.7 \pm 0.3$ & $0.48 \pm 0.01$ & $0.92 \pm 0.01$ & $150 \pm 8$ \\
Stroke & 8 & $2.6 \pm 0.1^{*}$ & $3.9 \pm 0.1^{*}$ & $6.2 \pm 0.2^{*}$ & $2.9 \pm 0.6^{*}$ & $0.83 \pm 0.08^{*, \S} 1.42 \pm 0.13^{*}$ & $279 \pm 16^{*, \S}$ \\
HF (early) or SD & 12 & $2.6 \pm 0.1^{*}$ & $4.0 \pm 0.1^{*}$ & $6.2 \pm 0.3^{*, \S}$ & $3.0 \pm 1.1^{*}$ & $0.86 \pm 0.11^{*, 8} 1.28 \pm 0.08^{*}$ & $270 \pm 19^{*, \S}$ \\
HF (late) & 21 & $2.4 \pm 0.1^{*}$ & $3.9 \pm 0.1^{*}$ & $6.9 \pm 0.1^{*}$ & $3.0 \pm 0.7^{*}$ & $0.70 \pm 0.02^{*}$ & $1.34 \pm 0.04^{*}$ & $245 \pm 21^{*}$ \\
\hline
\end{tabular}

Values are mean \pm SEM. HF (early) or SD, early developed heart failure rats or sudden death rats; HF (late), late developed heart failure rats. For other abbreviations see text. ${ }^{*} p<0.01 v s$. DR, ${ }^{8} p<0.05 v s$. HF (late).

Table 3. Indexes of Left Ventricular Geometry Obtained from M-Mode Echocardiography

\begin{tabular}{|c|c|c|c|c|c|c|c|c|}
\hline & Weeks & $\begin{array}{l}\text { PWTd } \\
(\mathrm{mm})\end{array}$ & $\begin{array}{l}\text { PWTs } \\
(\mathrm{mm})\end{array}$ & $\begin{array}{l}\text { EDD } \\
(\mathrm{mm})\end{array}$ & $\begin{array}{l}\text { ESD } \\
(\mathrm{mm})\end{array}$ & $\begin{array}{c}\text { RWT } \\
\text { (2PWTD/EDD) }\end{array}$ & $\begin{array}{l}\text { LVM } \\
(\mathrm{g})\end{array}$ & $\mathrm{ESD} / \mathrm{EDD}$ \\
\hline \multirow[t]{7}{*}{ DR } & 6 & $1.4 \pm 0.1$ & $2.4 \pm 0.1$ & $6.9 \pm 0.1$ & $3.4 \pm 0.1$ & $0.39 \pm 0.01$ & $0.57 \pm 0.01$ & $0.50 \pm 0.01$ \\
\hline & 8 & $1.6 \pm 0.1$ & $2.8 \pm 0.1$ & $7.3 \pm 0.1$ & $3.7 \pm 0.1$ & $0.43 \pm 0.01$ & $0.77 \pm 0.01$ & $0.51 \pm 0.01$ \\
\hline & 11 & $1.8 \pm 0.1$ & $2.9 \pm 0.1$ & $7.4 \pm 0.1$ & $3.7 \pm 0.1$ & $0.48 \pm 0.01$ & $0.92 \pm 0.01$ & $0.51 \pm 0.01$ \\
\hline & 13 & $1.8 \pm 0.1$ & $3.1 \pm 0.1$ & $7.7 \pm 0.1$ & $3.8 \pm 0.2$ & $0.47 \pm 0.01$ & $1.03 \pm 0.05$ & $0.49 \pm 0.01$ \\
\hline & 14 & $1.8 \pm 0.1$ & $3.1 \pm 0.1$ & $7.9 \pm 0.1$ & $3.9 \pm 0.2$ & $0.45 \pm 0.01$ & $1.04 \pm 0.04$ & $0.49 \pm 0.02$ \\
\hline & 15 & $1.8 \pm 0.1$ & $3.1 \pm 0.1$ & $7.8 \pm 0.1$ & $3.9 \pm 0.1$ & $0.46 \pm 0.01$ & $1.06 \pm 0.02$ & $0.50 \pm 0.01$ \\
\hline & 18 & $1.9 \pm 0.1$ & $3.1 \pm 0.1$ & $8.1 \pm 0.1$ & $4.2 \pm 0.2$ & $0.46 \pm 0.01$ & $1.16 \pm 0.09$ & $0.52 \pm 0.01$ \\
\hline \multirow[t]{7}{*}{ DS } & 6 & $1.3 \pm 0.1$ & $2.5 \pm 0.1$ & $6.8 \pm 0.1$ & $3.5 \pm 0.1$ & $0.40 \pm 0.01$ & $0.56 \pm 0.01$ & $0.52 \pm 0.01$ \\
\hline & 8 & $1.8 \pm 0.1^{*}$ & $3.4 \pm 0.1 *$ & $7.2 \pm 0.1$ & $3.0 \pm 0.1^{*}$ & $0.49 \pm 0.01 *$ & $0.90 \pm 0.01 *$ & $0.41 \pm 0.01 *$ \\
\hline & 11 & $2.5 \pm 0.1 *$ & $3.9 \pm 0.1 *$ & $6.9 \pm 0.1 *$ & $3.0 \pm 0.2 *$ & $0.70 \pm 0.02 *$ & $1.34 \pm 0.04 *$ & $0.43 \pm 0.02 *$ \\
\hline & 13 & $2.5 \pm 0.1^{*}$ & $3.8 \pm 0.1^{*}$ & $7.4 \pm 0.3$ & $3.7 \pm 0.3$ & $0.68 \pm 0.05 *$ & $1.56 \pm 0.04 *$ & $0.49 \pm 0.03$ \\
\hline & 14 & $2.4 \pm 0.1^{*}$ & $3.7 \pm 0.2 *$ & $8.0 \pm 0.2$ & $4.2 \pm 0.3$ & $0.61 \pm 0.02 *$ & $1.65 \pm 0.06^{*}$ & $0.53 \pm 0.04$ \\
\hline & 15 & $2.5 \pm 0.1^{*}$ & $3.7 \pm 0.1 *$ & $8.3 \pm 0.4$ & $4.7 \pm 0.5$ & $0.61 \pm 0.04 *$ & $1.83 \pm 0.13$ & $0.55 \pm 0.04$ \\
\hline & 18 & $2.4 \pm 0.1^{*}$ & $2.9 \pm 0.2$ & $9.0 \pm 0.4^{\S}$ & $6.7 \pm 0.6^{*}$ & $0.56 \pm 0.05 *$ & $1.98 \pm 0.09 *$ & $0.74 \pm 0.05 *$ \\
\hline \multicolumn{9}{|l|}{ ANOVA } \\
\hline Group & & 0.0001 & 0.0001 & 0.4609 & 0.0169 & 0.0001 & 0.0001 & 0.0393 \\
\hline Time & & 0.0001 & 0.0001 & 0.0001 & 0.0001 & 0.0001 & 0.0001 & 0.0001 \\
\hline Interaction & & 0.0001 & 0.0001 & 0.0044 & 0.0001 & 0.0001 & 0.0001 & 0.0001 \\
\hline
\end{tabular}

Values are mean \pm SEM. For abbreviations see text. Main effects were assessed using repearted measured ANOVA. Between group and within group comparisons at each time point were done using one factor ANOVA followed by Fisher's protected least significant defference test. ${ }^{*} p<0.001,{ }^{\S} p<0.05 v s$. DR at same time point. 

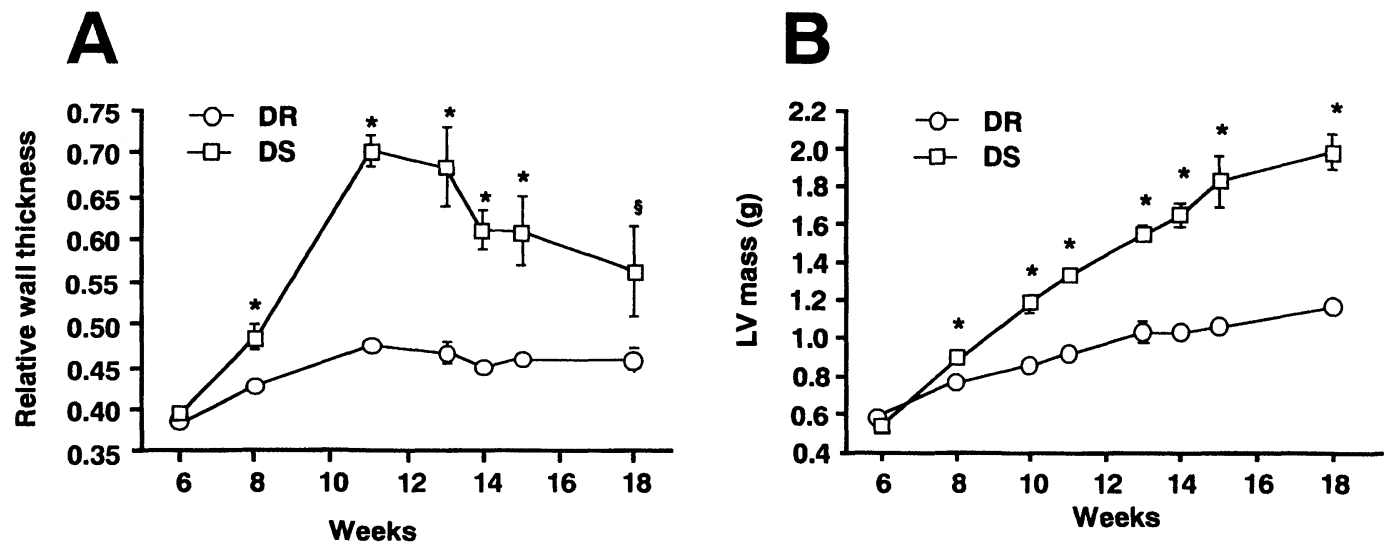

Fig. 4. Serial changes in relative wall thickness $(A)$ and $L V$ mass $(B)$ in Dahl salt-sensitive $(D S)$ rats $(\mathrm{n}=18)$ and Dahl salt-resistant $(D R)(\mathrm{n}=21)$ rats at the ages of $6,8,11,13,14,15$, and 18 weeks. ${ }^{*} \mathrm{p}<0.001$ vs. $D R$.

Table 4. Indexes of Left Ventricular Systolic Function Obtained from M-Mode Echocardiography in Dahl Rats

\begin{tabular}{|c|c|c|c|c|c|c|c|}
\hline & Weeks & $\begin{array}{l}\mathrm{EF} \\
(\%) \\
\end{array}$ & $\begin{array}{c}\% \text { PWT } \\
(\%)\end{array}$ & $\begin{array}{l}\text { FS } \\
(\%)\end{array}$ & $\begin{array}{c}\mathrm{mFS} \\
(\%)\end{array}$ & $\begin{array}{c}\text { ESSmwc } \\
\left(\mathrm{kdyne} / \mathrm{cm}^{2}\right)\end{array}$ & $\begin{array}{c}\text { ESSmwm } \\
\left(\mathrm{kdyne} / \mathrm{cm}^{2}\right)\end{array}$ \\
\hline \multirow[t]{7}{*}{ DR } & 6 & $87.4 \pm 0.9$ & $80.8 \pm 2.4$ & $50.1 \pm 1.2$ & $28.6 \pm 0.8$ & $90.9 \pm 4.1$ & $85.7 \pm 3.4$ \\
\hline & 8 & $86.4 \pm 0.8$ & $75.8 \pm 1.5$ & $48.7 \pm 1.0$ & $26.6 \pm 0.7$ & $84.7 \pm 2.6$ & $76.9 \pm 4.2$ \\
\hline & 11 & $86.9 \pm 0.5$ & $70.2 \pm 1.9$ & $49.4 \pm 0.7$ & $27.4 \pm 0.3$ & $85.0 \pm 2.8$ & $80.9 \pm 1.8$ \\
\hline & 13 & $87.7 \pm 1.0$ & $73.9 \pm 3.5$ & $50.6 \pm 1.3$ & $27.0 \pm 0.7$ & $80.2 \pm 4.0$ & $78.8 \pm 2.4$ \\
\hline & 14 & $87.8 \pm 1.1$ & $77.1 \pm 3.0$ & $50.8 \pm 1.5$ & $27.3 \pm 0.7$ & $80.3 \pm 5.1$ & $78.3 \pm 3.1$ \\
\hline & 15 & $87.7 \pm 0.7$ & $69.7 \pm 2.5$ & $50.4 \pm 1.0$ & $27.9 \pm 0.6$ & $82.1 \pm 4.6$ & $80.1 \pm 2.1$ \\
\hline & 18 & $85.8 \pm 1.1$ & $66.7 \pm 2.4$ & $48.2 \pm 1.4$ & $26.7 \pm 0.7$ & $98.6 \pm 6.1$ & $88.8 \pm 4.0$ \\
\hline \multirow[t]{7}{*}{ DS } & 6 & $86.2 \pm 0.9$ & $83.6 \pm 1.3$ & $48.5 \pm 1.2$ & $26.6 \pm 0.8$ & $98.2 \pm 3.6$ & $91.2 \pm 3.3$ \\
\hline & 8 & $93.0 \pm 0.6^{*}$ & $94.4 \pm 3.3 *$ & $58.7 \pm 1.1 *$ & $29.1 \pm 0.6^{*}$ & $67.8 \pm 4.7 *$ & $69.3 \pm 2.8$ \\
\hline & 11 & $91.0 \pm 1.1^{\S}$ & $62.6 \pm 2.0$ & $56.5 \pm 1.3 *$ & $25.6 \pm 1.2$ & $67.4 \pm 6.8 *$ & $90.6 \pm 4.6^{\S}$ \\
\hline & 13 & $86.7 \pm 2.1$ & $52.5 \pm 4.6^{*}$ & $50.7 \pm 2.2$ & $24.4 \pm 1.0^{\S}$ & $103.8 \pm 13.1$ & $113.5 \pm 7.4 *$ \\
\hline & 14 & $84.2 \pm 3.5$ & $51.2 \pm 5.2 *$ & $49.0 \pm 3.6$ & $24.3 \pm 1.8^{\S}$ & $122.2 \pm 18.1^{*}$ & $123.0 \pm 11.5^{*}$ \\
\hline & 15 & $80.9 \pm 3.4$ & $49.7 \pm 3.1 *$ & $46.3 \pm 3.9$ & $22.6 \pm 2.0^{\S}$ & $129.4 \pm 26.1^{*}$ & $156.1 \pm 19.1^{*}$ \\
\hline & 18 & $55.9 \pm 7.0 *$ & $20.1 \pm 3.6^{*}$ & $25.7 \pm 4.5 *$ & $15.4 \pm 2.5 *$ & $218.1 \pm 30.3$ & $168.3 \pm 19.5 *$ \\
\hline \multicolumn{8}{|c|}{ ANOVA } \\
\hline & & 0.0001 & 0.0001 & 0.0393 & 0.0001 & 0.0001 & 0.0001 \\
\hline $\mathrm{Ti}$ & & 0.0001 & 0.0001 & 0.0001 & 0.0001 & 0.0001 & 0.0001 \\
\hline & & 0.0001 & 0.0001 & 0.0001 & 0.0001 & 0.0001 & 0.0001 \\
\hline
\end{tabular}

Values are mean \pm SEM. For abbreviations see text. ${ }^{*} p<0.001,{ }^{\S} p<0.05 v s$. DR at same time point.

than in DR rats. There was no significant difference in LV mass among the 3 groups of DS rats. However, RWT and systolic blood pressure were greater in rats with stroke and early-developed heart failure than in DS rats with late developed heart failure.

\section{Serial Changes of LV Geometry in the Development of Hypertension}

The time course of LV geometric changes in DR and DS rats after being administered the $8 \%$ salt-diet is shown in
Table 3. PWTd and PWTs in DS rats progressively increased, reaching a plateau at 11 weeks of age. PWTd in DS rats remained at the plateau level until 18 weeks. In contrast, PWTs after 11 weeks gradually decreased, and at 18 weeks of age was lower than that of DR rats.

Concerning the alterations in LV chamber size, EDD increased at the same rate in DS rats as in DR rats at the early stage of hypertension. After the age of 14 weeks, EDD was greater in DS than in DR rats. The ESD of DR rats remained almost constant between the 8 and 15 weeks of age. However, the ESD of DS rats gradually in- 

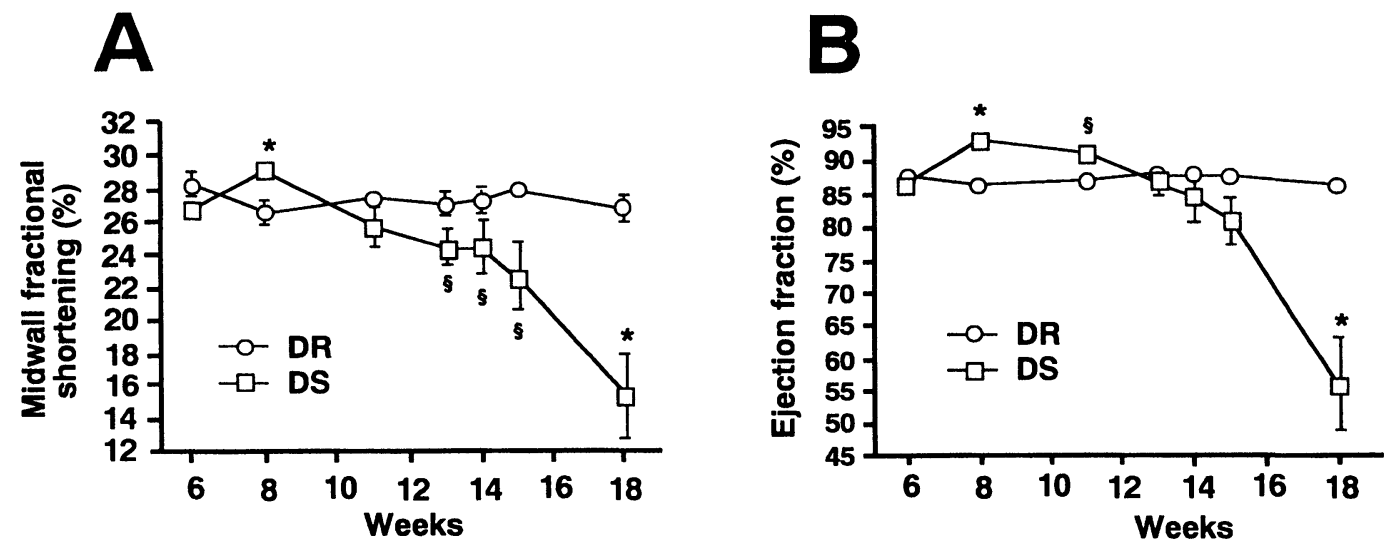

Fig. 5. Time course of the $L V$ systolic function obtained from M-mode echocardiography in Dahl salt-resistant (DR) (n= 21) and Dahl salt-sensitive $(D S)(\mathrm{n}=18)$ rats at the ages of $6,8,11,13,14,15$, and 18 weeks. A: midwall fractional shortening, B: ejection fraction. ${ }^{*} \mathrm{p}<0.001,{ }^{\S} \mathrm{p}<0.05$ vs. DR rats.
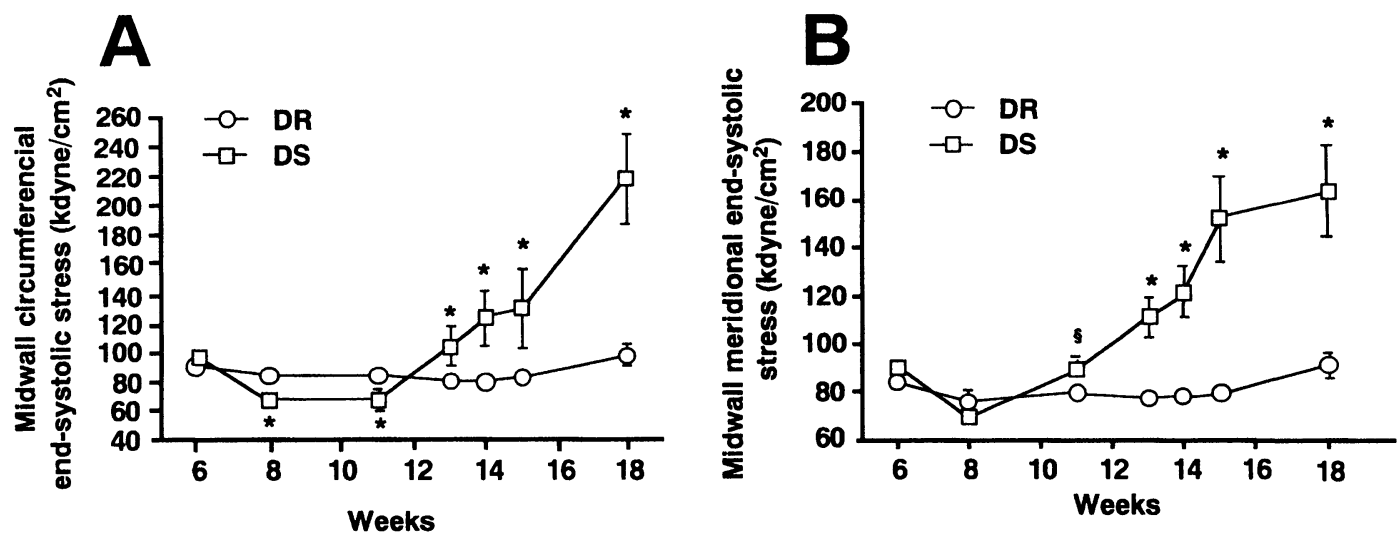

Fig. 6. Time course of wall stress in Dahl salt-sensitive (DS) and Dahl salt-resistant(DR) rats at the ages of 6, 8, 11, 13, 14,15 , and 18 weeks after feeding an $8 \% \mathrm{NaCl}$ diet. A: midwall circumferential end-systolic wall stress, B: midwall meridional end-systolic wall stress. ${ }^{*} \mathrm{p}<0.001,{ }^{\S} \mathrm{p}<0.05$ vs. $D R$.

creased from 11-18 weeks.

Figure 4 shows the serial changes in RWT (A) and LV mass (B). RWT in DR rats progressively increased, reaching a plateau at 11 weeks. That in DS rats also progressively increased to the age of 11 weeks, after it gradually declined. LV mass continued to increase throughout the study in both DR and DS rats, and LV mass was always greater in DS than in DR rats.

\section{Serial Changes in LV Function Identified by Echocar- diography}

Table 4 and Fig. 5 show the time course of the LV systolic function obtained from M-mode echocardiograms in DR and DS rats. In DR rats EF, \% PWT, FS, and mFS remained constant throughout the study period. In contrast, each of these parameters in DS rats followed the same pattern: between 8 and 11 weeks levels were elevated in comparison to the age-matched DR rats. However, after 11 weeks, their values progressively decreased, and, particularly, \%PWT and mFS between 13 and 18 weeks were significantly less than those of age-matched DR rats.

Figure 6 shows the time course of ESSmwc (A) and ESSmwm (B) in DR and DS rats. In DR rats, both ESSmwc and ESSmwm were almost constant for rats of all ages observed in the study period. In DS rats, both ESSmwc and ESSmwm were the same or lower when compared to the DR rats matched in age. However, after the age of 11-14 weeks, these parameters continued to increase corresponding to the increase in age. 
Table 5. Time Course of in Vivo Hemodynamics in DR and DS Rats during the Salt Loading

\begin{tabular}{lrrccccc}
\hline & Weeks & $n$ & $\begin{array}{c}\text { LVSP } \\
(\mathrm{mmHg})\end{array}$ & $\begin{array}{c}\text { LVEDP } \\
(\mathrm{mmHg})\end{array}$ & $\begin{array}{c}+\mathrm{d} P / \mathrm{d} t \\
(\mathrm{mmHg} / \mathrm{s})\end{array}$ & $\begin{array}{c}-\mathrm{d} P / \mathrm{d} t \\
(\mathrm{mmHg} / \mathrm{s})\end{array}$ & $\begin{array}{c}\mathrm{d} P / \mathrm{d} t / P \\
(1 / \mathrm{s})\end{array}$ \\
\hline DR & 6 & 10 & $136.0 \pm 3.8$ & $5.4 \pm 0.7$ & $7,511 \pm 436$ & $6,244 \pm 659$ & $250.0 \pm 0.2$ \\
& 8 & 9 & $137.0 \pm 4.2$ & $5.6 \pm 0.8$ & $7,055 \pm 662$ & $6,611 \pm 582$ & $243.0 \pm 5.6$ \\
& 11 & 10 & $149.0 \pm 2.6$ & $5.9 \pm 0.5$ & $7,590 \pm 466$ & $6,375 \pm 374$ & $249.0 \pm 2.3$ \\
& 14 & 10 & $148.0 \pm 2.8$ & $5.3 \pm 0.6$ & $7,580 \pm 450$ & $6,620 \pm 550$ & $245.0 \pm 2.8$ \\
& 18 & 10 & $154.0 \pm 3.0$ & $6.1 \pm 0.8$ & $7,680 \pm 568$ & $6,990 \pm 564$ & $239.0 \pm 3.0$ \\
DS & 6 & 10 & $142.0 \pm 3.9$ & $5.8 \pm 0.6$ & $7,890 \pm 295$ & $6,330 \pm 198$ & $249.0 \pm 1.8$ \\
& 8 & 9 & $168.0 \pm 3.7^{*}$ & $7.3 \pm 0.6$ & $9,800 \pm 839^{*}$ & $8,175 \pm 422^{*}$ & $229.0 \pm 13.7$ \\
& 11 & 10 & $217.0 \pm 7.8^{*}$ & $7.8 \pm 1.0$ & $8,200 \pm 826$ & $6,350 \pm 572$ & $238.0 \pm 10.8$ \\
& 14 & 9 & $205.0 \pm 9.2^{*}$ & $12.5 \pm 4.9 \S$ & $7,200 \pm 700$ & $6,000 \pm 560$ & $222.0 \pm 7.5$ \\
& 18 & 8 & $185.0 \pm 16.9^{\S}$ & $33.0 \pm 8.8$ & $5,400 \pm 1,000 *$ & $3,233 \pm 948 *$ & $154.0 \pm 4.3^{*}$ \\
ANOVA & & & & & & & \\
Group & & & 0.0001 & 0.0001 & 0.6778 & 0.089 & 0.0001 \\
Time & & 0.0001 & 0.0001 & 0.4218 & 0.0266 & 0.0001 \\
Interaction & & 0.0001 & 0.0001 & 0.0001 & 0.0001 & 0.0001 \\
\hline
\end{tabular}

Values are mean \pm SEM. For abbreviations see text. In vivo hemodynamics were recorded just after the echocardiographic examination. ${ }^{*} p<0.01,{ }^{\S} p<0.05 v s$. DR in the same time point.

\section{Serial Changes in Hemodynamic Parameters Obtained from Cardiac Catheterization}

In vivo hemodynamic measurements obtained through the catheter in DR and DS rats at the age of $6,8,11,14$, or 18 weeks are shown in Table 5 . The LV end-diastolic pressure (LVEDP) in DS rats remained normal at the age of 11 weeks. However, it was significantly increased after 14 weeks of age, and at 18 weeks LVEDP increased to $33.0 \pm 8.8 \mathrm{mmHg}$. The patterns of $+\mathrm{d} P / \mathrm{d} t$ and $-\mathrm{d} P / \mathrm{d} t$, which changed according to the time course, were almost the same, increaseing significantly at 8 weeks of age compared with age-matched DR rats, and gradually decreasing over time. There was a cross-point of $+\mathrm{d} P / \mathrm{d} t$ or $-\mathrm{d} P / \mathrm{d} t$ near 13 weeks between DS and DR rats. Both $+\mathrm{d} P / \mathrm{d} t$ and $-\mathrm{d} P / \mathrm{d} t$ markedly decreased at 18 weeks of age. The $\mathrm{d} P / \mathrm{d} t / P$, which reflects changes in $\mathrm{d} P / \mathrm{d} t$ independent of pressure, also markedly decreased at 18 weeks in DS rats.

\section{Discussion}

In this study, we observed the cardiovascular events associated with the progression of hypertension in DS rats and confirmed the cause of death by autopsy. In addition, we studied serial changes in LV geometry and function from the development of hypertension to heart failure. Though approximately $60 \%$ of deaths in DS rats were due to heart failure, death in the early stages of hypertension was usually due to either sudden death or stroke. High RWT values and small EDD were predictive factors of sudden death and stroke, whereas LV mass was not.
Concerning the change in LV geometric patterns, LV mass continued to increase to 18 weeks. RWT increased or remained high to 13 weeks, after which it progressively decreased. In contrast, LV chamber function was hyperdynamic at the early stage (between 8 and 11 weeks), and remained almost normal between 11 and 13 weeks. Then, after 13 weeks, all hemodynamic variables of DS rats progressively deteriorated, and all DS rats died of heart failure. Thus, our study indicates that cardiovascular events associated with the progression of hypertension are different according to the stage of hypertension and that predictable risk factors for cardiovascular events are also different.

\section{Cardiovascular Events Associated with the Progres- sion of Hypertension}

Survival analysis in this study showed that the incidence of death in the DS rats was $57.6 \%$ from heart failure, $27.3 \%$ from stroke, and $15.2 \%$ from sudden death. However, the incidence of death before 14 weeks of age was $27.3 \%$ from stroke, $24.2 \%$ from heart failure, and $15.2 \%$ from sudden death. Notably, death in the early stage of hypertension was almost always due to sudden death or stroke. In view of these findings regarding cardiovascular events, the present study clearly elucidates two points. First, there is a marked difference in the stages of hypertension at which stroke and heart failure occur. Stroke usually appears earlier than heart failure. Second, sudden death is an important cause of death, at least in this hypertensive model.

Hemodynamic characteristics identified for DS rats with 
stroke and sudden death at the age of 11 weeks include small EDD, high RWT values, and high blood pressures (Table 2). However, there was no significant difference in LV mass between these rats and DS rats with late-developing heart failure. It is well known that increased LV mass predicts cardiovascular events $(1,3,24)$. However, our results indicate that a small EDD and a high RWT value are stronger predictors of cardiovascular events than in LV mass. Our findings also support other studies showing that RWT is an important and independent risk factor for cardiovascular events $(2,24)$.

\section{Serial Changes in the LV Geometric Pattern}

The pattern of LV geometric change in DS rats associated with the progression of systolic blood pressure was considerably different at various stages of hypertension. First, the geometric pattern at the early stage of hypertension in DS rats between 8 and 11 weeks was characterized by smaller EDD and ESD, thicker PWTd, and greater RWT and LV mass than age-matched DR rats. Second, at the stage from 11-13 weeks, EDD and ESD in DS rats were identical to those of age-matched DR rats. PWTd and RWT remained unchanged compared to the earlier stage, but LV mass continued to increase. Third, after 13 weeks, both EDD and ESD tended to dilate and PWTd remained unchanged; thus, RWT gradually decreased. However, even at this stage, the LV mass gradually increased. In view of these findings, we may conclude, regarding the LV morphological adaptation associated with hypertension, that there is a limit to increasing LV wall thickness in response to increases in afterload. This finding suggests that the increase in RWT is also limited, although LV mass has no limit. In this sense, RWT may more strongly predict cardiovascular morbidity and mortality than does LV mass. The survival analysis of our data may support this theory.

\section{The Progression from LV Dysfunction to Heart Fail- ure}

The LV function of DS rats was considerably different according to the LV geometric pattern. At the early stage of hypertension, between 8 and 11 weeks of age, the LV function was characterized by a supernormal state, reflected by greater $\mathrm{FS}, \mathrm{mFS}, \mathrm{EF}, \% \mathrm{PWT},+\mathrm{d} P / \mathrm{d} t$, and $-\mathrm{d} P / \mathrm{d} t$. In addition, the ESSmwc of DS rats was lower than that of DR rats, despite a marked elevation of systolic blood pressure. The exact mechanism for the hypercontractile state of this stage remains to be determined.

At the stage from 11-13 weeks, the LV function of DS rats remained almost identical to that of age-matched DR rats. However, the mFS and \% PWT were lower than those of age-matched DR rats. In addition, both ESSmwc and ESSmwm were already elevated in DS rats, indicat- ing that mild myocardial dysfunction already existed at that time in the rats. Thus, the early stage of concentric LV hypertrophy that has been classically described as "compensated" may in fact be characterized by mild abnormalities of myocardial contractility that are not detected using conventional measurements of endocardial shortening or ejection performance. The sensitivity of parameters used in evaluating $L V$ function should be determined in the future.

After 13 weeks, all hemodynamic variables of DS rats progressively deteriorated, and all DS rats had died of heart failure by the end of 19 weeks. A gradual decrease in systolic blood pressure after 13 weeks in DS rats may be related to the decrease in stroke volume. The elevation of ESSmwm is the earliest detectable change in all of the hemodynamic variables, and this elevation may be closely related to the decrease in LV contractility associated with LV hypertrophy. We have recently reported that left ventricular remodeling closely reflects myocytes function (25). Thus, the elevation of ESSmwm may reflect myocytes dysfunction. The exact mechanism for this decrease in LV contractility is not fully understood. One of the most important mechanisms, however, may be related to the reduction of coronary blood flow reserve in hypertensive patients with LV hypertrophy (26-30). We have reported that coronary blood flow increases parallel to the increase in LV mass, but coronary blood flow per $100 \mathrm{~g}$ of myocardium decreases according to the increase in LV mass (31). This myocardial ischemia may induce LV dysfunction, which can lead to heart failure. In fact, the results of our recent study also indicate that there is a very close relationship between coronary blood flow per $100 \mathrm{~g}$ of myocardium and LV systolic and diastolic functions (32). Another possible mechanism is increased interstitial collagen and fibrosis (33). These may also result in myocardial cell death. A substantial myocardial cell death or myocytes dysfunction induces a decrease in cardiac performance, which results in left ventricular dilatation. This dilatation results in an increase in wall stress, which deteriorates fiber shortening and ejection and can result in heart failure. In addition, the LV dysfunction associated with hypertensive heart failure can seldom be returned to normal by long-term antihypertensive therapy (34). Increasing afterload by hypertension triggers this vicious cycle. Thus, in the clinical setting we must strictly control blood pressure.

\section{Comparison of the Sensitivity of Hemodynamic Param- eters}

In the present study we compared the sensitivity of hemodynamic parameters as determined from the endocardial and midwall portions of the ventricular wall to evaluate the LV condition in those suffering from hypertension. Our results indicate that $\mathrm{mFS}$ can indicate abnor- 
malities earlier than FS and EF. Shimizu et al. and de Simone et al. have reported that mFS is more sensitive than FS for evaluating hypertrophied hearts, and that midwall determination is less dependent on LV geometry $(20,35)$. Gaaschetal $(21)$ have also reported as the result of an experimental study that $\mathrm{mFS}$ can indicate depressed myocardial contractility due to chronic LV pressure overload in dogs earlier than abnormalities of endocardial shortening. Thus, we should be careful when LV functions are evaluated using hemodynamic parameters gained from endocardial tracing.

\section{Study Limitations}

Our study has a few limitations. One limitation is the accuracy of echocardiographic measurements. Although high-frequency ultrasound can identify separate objects that are $0.2 \mathrm{~mm}$ apart, there is some difficulty in measuring cardiac dimension and wall thickness echocardiographically in rats. According to Fig. 1 and the study of Inoko et al. (13), a comparison of LV masses calculated by the cube-function formula from echocardiographic measurements with those observed at autopsy has shown a linear correlation with narrow deviations in every stage of DS and DR rats. These findings may support our echocardiographically determined data.

\section{References}

1. Levy D, Garrison RJ, Savage DD, Kannel WB, Castelli WP: Prognostic implications of echocardiographically determined left ventricular mass in the Framingham Heart Study. N Engl J Med 1990; 322: 1561-1566.

2. Koren MJ, Devereux RB, Casale PN, Savage DD, Laragh $\mathrm{JH}$ : Relation of left ventricualr mass and geometry to morbidity and mortality in uncomplicated essential hypertension. Ann Intern Med 1991; 114: 345352.

3. Hamada M, Hiwada K, Kokubu T: Clinical significance of systolic time intervals in hypertensive patients. Eur Heart J 1990; 11 (Suppl I): 105-113.

4. Shigematsu $\mathbf{Y}$, Hamada $\mathbf{M}$, Mukai $\mathbf{M}$, Matsuoka $\mathrm{H}$, Sumimoto T, Hiwada K: Clinical evidence for an association between left ventricular geometric adaptation and extracardiac target organ damage in essential hypertension. J Hypertens 1995; 13: 155-160.

5. Devereux RB, de Simone G, Pickering TG, Schwartz JE, Roman MJ: Relation of left ventricular midwall function to cardiovascular risk factiors and arterial structure and function. Hypertension 1998; 31: 929-936.

6. Randhawa AK, Singal PK: Pressure overload-induced cardiac hypertrophy with and without dilation. J Am Coll Cardiol 1992; 20: 1569-1575.

7. Siri FM, Nordin SM, Factor SM, Sonnenblick E, Aronson R: Compensatory hypertrophy and failure in gradual pressure-overloaded guinea pig heart. Am J Physiol
1989; 257: H1016-H1024.

8. Capasso JM, Palackal T, Olivetti G, Anversa P: Left ventricular failure induced by long-term hypertension in rats. Circ Res 1990; 66: 1400-1412.

9. Pfeffer MA, Pfeffer JM, Fishbein MC, et al.: Myocardial infarct size and ventricular function in rats. Circ Res 1979; 44: 503-512.

10. Litwin SE, Katz SE, Weinberg EO, Lorell BH, Aurigemma GP, Douglas PS: Serial EchocardiographicDoppler assessment of left ventricular geometry and function in rats with pressure-overload hypertrophy: chronic angiotensin-converting enzyme inhibition attenuates the transition to heart failure. Circulation 1995; 91: 2642-2654.

11. Feldman AM, Weinberg EO, Ray PE, Lorell BH: Selective changes in cardiac gene expression during compensated hypertrophy and the transition to cardiac decompensation in rats with chronic aortic banding. Circ Res 1993; 73: 184-192.

12. Bing OHL, Brooks WW, Robinson KG, Slawsky MT, Hayes JA, Litwin SE: The spontaneously hypertensive rat as a model of the transition from compensated left ventricular hypertrophy to failure. J Mol Cell Cardiol 1995: 27: 383-396.

13. Inoko M, Kihara Y, Moril I, FujiwaraH, SasayamaS: Transition from compensatory hypertrophy to dilated, failing left ventricules in Dahl salt-sensitive rats. Am J Physiol 1994; 267: H2471-H2482.

14. Dahl LK, Knudsen KD, Herin MA, Leitl GJ: Effects of chronic excess salt ingestion: modification of experimental hypertension in the rat by variations in the diet. Circ Res 1968; 22: 11-18.

15. Pfeffer MA, Pfeffer JM, Mirsky I, Iwai J: Cardiac hypertrophy and performance of Dahl hypertensive rats on graded salt diets. Hypertension 1984; 6: 475-481.

16. von Lutterotti N, Camargo MJF, Compbell WG et al.: Angiotensin II receptor antagonist delay renal damage and stroke in salt-loaded dahl salt-sensitive rats. $J$ Hypertens 1992; 10: 949-957.

17. Sahn DJ, DeMaria A, Kisslo J, Weyman A: Recommendations regarding quantitation in M-mode echocardiography: results of a survey of echocardiographic measurements. Circulation 1978; 58: 1072-1083.

18. de Somone G, Wallerson DC, Volpe M, Devereux RB: Echocardiographic measurement of left ventricular mass and volume in normotensive and hypertensive rats. Necropsy validation. Am J Hypertens 1990; 3: 688-696.

19. Pawlusch DG, Moore RL, Musch TI, et al: Echocardiographic evaluation of size, function and mass of normal and hypertrophied rat ventricles. J Appl Physiol 1993; 74: 2598-2630.

20. Shimizu G, Hirota Y, Kita Y, Kawamura K, Saito T, Gaasch WH: Left ventricular midwall mechanics in systemic arterial hypertension: myocardial function is depressed in pressure-overload hypertrophy. Circulation 1991; 83: 1676-1684.

21. Gaasch WH, Bile MR, Hoshino PK, Apstein CS, Blaustein AS: Stress-shortening relations and myocardial blood flow in compensated and failing canine hearts with pressure-overload hypertrophy. Circulation 1989; 79: 
872-883.

22. de Simone G, Devereux RB, Roman MJ, et al.: Assessment of left ventricular function by the mid-wall fractional shortening end-systolic stress relation in human hypertension. J Am Coll Cardiol 1994; 23: 1444-1451.

23. Graham TP Jr, Franklin RG, Wyse RKH, Gooch V, Deanfield JE: Left ventricular wall stress and contractile function in childhood: normal values and comparison of Fontan repair versus palliation only in patients with tricuspid atresia. Circulation 1986; 74 (Suppl I): I-61-I-69.

24. Shigematsu Y, Hamada M, Ohtsuka T, et al: Left ventricular geometry as an independent predictor for extracardiac target organ damage in essential hypertension. Am J Hypertens 1998; 11: 1171-1177.

25. Ohtsuka T, Suzuki M, Hamada M, Hiwada K: Cardiomyocyte functions couple with left ventricular geometric patterns in hypertension. Hypertens Res 2000; 23: 345-351.

26. Hofmann JIE: A critical view of coronary reserve. Circulation 1987; 75 (Suppl I): I-6-I-11.

27. Houghton JL, Frank MJ, Carr AA, von Dohlen TW, Prisant LM: Relations among impaired coronary flow reserve, left ventricular hypertrophy and thallium perfusion defects in hypertensive patients without obstructive coronary artery disease. J Am Coll Cardiol 1990; 15: 4351.

28. Antony I, Nitenberg A, Foult J-M, Aptecar E: Coronary vasodilator reserve in untreated and treated hypertensive patients with and without left ventricular hyper- trophy. J Am Coll Cardiol 1993; 22: 514-520.

29. Motz W, Strauer BE: Improvement of coronary flow reserve after long-term therapy with enalapril. Hypertension 1996; 27: 1031-1038.

30. Kozàkovà M, Palombo $\mathrm{C}$, Pratali L, Pittella G, Galetta F, L'Abbate A: Mechanisms of coronary flow reserve impairment in human hypertension: an integrated approach by transthoracic and transesophageal echocardiography. Hypertension 1997; 29: 551-559.

31. Hamada M, Kuwahara T, Shigematsu Y, et al: Relation between coronary blood flow and left ventricular mass in hypertension: noninvasive quantification of coronary blood flow by thallium-201 myocardial scintigraphy. Hypertens Res 1998; 21: 227-234.

32. Sasaki O, Hamada M, Hiwada K: Effect of coronary blood flow on left ventricular function in essential hypertensive patients. Hypertens Res 2000; 23: 239-245.

33. Weber KT, Brilla CG: Pathological hypertrophy and cardiac interstitium: fibrosis and renin-angiotensin-aldosterone system. Circulation 1991: 83: 1849-1865.

34. Hamada M, Ohtani T, Suzuki M, et al: Is left ventricular dysfunction in hypertensive patients with heart failure normalized by long-term antihypertensive therapy? $J$ Cardiol 1993; 23: 165-175.

35. Shimizu G, Zile MR, Blaustein AS, Gaasch WH: Left ventricular chamber filling and midwall fiber lengthening in patients with left ventricular hypertrophy: overestimation of fiber velocities by conventional midwall measurements. Circulation 1985; 71: 266-272. 